\title{
On the corrections to the Casimir effect depending on the resolution of measurement
}

\author{
M.V.Altaisky \\ Joint Institute for Nuclear Research, Joliot Curie 6, \\ Dubna, 141980, Russia; and Space Research Institute RAS, \\ Profsoyuznaya 84/32, Moscow, 117997, Russid* \\ N.E.Kaputkina \\ National University of Science and Technology "MISiS", \\ Leninsky prospect 4, Moscow, 119049, Russid
}

(Dated: Mar 30, 2011: Revised July 16, 2011)

\begin{abstract}
The Casimir force $\mathcal{F}=-\frac{\pi^{2} \hbar c}{240 a^{4}}$, which attracts to each other two perfectly conducting parallel plates separated by the distance $a$ in vacuum, is one of the blueprints of the reality of vacuum fluctuations. Following the recent conjecture, that quantum fields should be described in terms of the fields depending on the resolution of measurement, rather than the position alone (M.V.Altaisky, Phys. Rev. D 81(2010)125003), we derive the correction to the Casimir energy depending on the ratio of the plate displacement amplitude to the distance between plates.
\end{abstract}

PACS numbers: 12.20.Ds, 12.20.Fv

Keywords: Casimir effect, quantum electrodynamics, regularization

The Casimir force is the result of the dif- bounded between conducting walls is said to ference of the vacuum zero point energy of the be dimensionally quantized, while in the lattwo different configurations: the rectangular ter case the frequency spectrum is continuvolume $L_{x} \times L_{y} \times a$ bounded by two paral- ous. The energy difference between these two lel conducting walls, and that of the same configurations cannot be measured directly, volume not bounded by conducting walls. but it varies with the variation of the gap In the former case, the electromagnetic field $a$, and this variation can be measured as the

*Electronic address: altaisky@mx.iki.rssi.ru Casimir force.

†Electronic address: nataly@misis.ru

In 1948 Casimir conjected that the force 
between two parallel conducting planes depends only on two universal constants, $\hbar$ and $c$, and the distance between the plates $a[1]$. The first attempt to measure the Casimir force has been undertaken in 1958 [2]. Later the Casimir force have been measured with an atomic force microscope [3, 4]. A lot of studies related to the Casimir effect are being carried out in different branches of nanomechanics and photonics now, see e.g. [5, 6] and references therein for recent review.

In the dimensionally quantized case the zero point energy of the electromagnetic field between conducting plates is

$$
\begin{aligned}
E_{Q} & =\frac{\hbar c}{2} \sum_{\alpha}\left|k_{\alpha}\right|=\frac{\hbar c}{2} \int \frac{L_{x} L_{y} d k_{\|}^{2}}{(2 \pi)^{2}}\left[\left|k_{\|}\right|+\right. \\
& \left.+2 \sum_{n=1}^{\infty}\left(k_{\|}^{2}+\frac{\pi^{2} n^{2}}{a^{2}}\right)^{1 / 2}\right]
\end{aligned}
$$

where factor 2 with the sum over discrete spectra accounts for two possible polarizations of the electromagnetic field; $k_{\|} \equiv$ $\left(k_{x}, k_{y}\right)$. The energy of the same field free of any boundary conditions is expressed as integral over continuous spectra $E_{0}=\frac{\hbar c}{2} \int \frac{L_{x} L_{y} d k_{\|}^{2}}{(2 \pi)^{2}} \int_{-\infty}^{\infty} 2 \frac{d k_{z}}{(2 \pi)}\left(k_{\|}^{2}+k_{z}^{2}\right)^{1 / 2}$.

Both integrals (12) are evidently infinite, but their difference

$$
\mathcal{E}=\frac{E_{Q}-E_{0}}{L_{x} L_{y}}
$$

known as the Casimir energy, can be regularized if the r.h.s. of the equations (1, 2) are multiplied by some cutoff function $f(k)$, such that $f(0)=1$ and $f\left(k \gg \frac{1}{a_{0}}\right) \rightarrow 0$, where $1 / a_{0}$ is the inverse size of atom. This specific choice accounts for the fact, that the walls are metallic plates made of real atoms, rather than of an ideal conductor.

Such choice of the cutoff function $f$ is practically appropriate, but does not relate the calculated ultra-violet infinities to what is really measured at the finite scale of measurement $\delta$, see Fig. 1. The conjecture, relat-

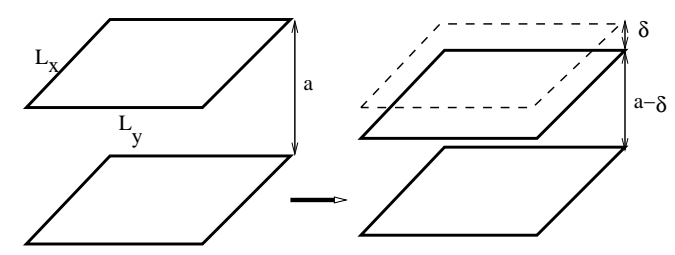

FIG. 1: Shift of the upper conducting wall from $z=a$ to $z=a-\delta$ changes the Casimir energy from $\mathcal{E}(a, \delta)$ to $\mathcal{E}(a-\delta, \delta)$

ing the quantum fields to the aperture function of the measurement taken with resolution $\delta$ was given in [7]. The aperture function $g(x)=-x e^{-x^{2} / 2}$ leads in one-loop approximation, up to appropriate rescaling, to the cutoff function

$$
f(k)=e^{-4 \delta^{2} k^{2}}
$$

After the choice (44) the regularized Casimir 
energy is (see $§ 3.2 .4$ of [8] ):

$$
\begin{aligned}
\mathcal{E} & =\frac{\hbar c \pi^{2}}{4 a^{3}}\left[\frac{F(0)}{2}+F(1)+F(2)+\ldots\right. \\
& \left.-\int_{0}^{\infty} d n F(n)\right], \\
F(n) & =\int_{0}^{\infty} d u \sqrt{u+n^{2}} e^{-4 \pi^{2} \frac{\delta^{2}}{a^{2}}\left(u+n^{2}\right)} \\
& =\frac{\sqrt{\pi}}{2\left(\frac{2 \pi \delta}{a}\right)^{3}}\left[1-\operatorname{erf}\left(\frac{2 \pi \delta}{a} n\right)+\right. \\
& \left.+4 \sqrt{\pi} \exp \left(-\left(\frac{2 \pi \delta}{a}\right)^{2} n^{2}\right) \frac{\delta}{a} n\right] .
\end{aligned}
$$

The difference between the sum and the integral in (5) is evaluated by Euler-Maclaurin formula

$$
\begin{array}{r}
\frac{1}{2} F(0)+F(1)+\ldots-\int_{0}^{\infty} d n F(n)= \\
\quad=-\frac{1}{2 !} B_{2} F^{\prime}(0)-\frac{1}{4 !} B_{4} F^{\prime \prime \prime}(0)-\ldots,
\end{array}
$$

where $B_{n}$ are the Bernoulli numbers. This gives the corrections to the Casimir energy

$$
\begin{aligned}
\mathcal{E}(a, \delta) & =-\frac{\hbar c \pi^{2}}{720 a^{3}}\left[1+\frac{2}{7}\left(\frac{2 \pi \delta}{a}\right)^{2}+\right. \\
& \left.+\frac{3}{28}\left(\frac{2 \pi \delta}{a}\right)^{4}+\ldots\right]
\end{aligned}
$$

and the Casimir force

$$
\begin{aligned}
\mathcal{F}(a, \delta) & =-\frac{\hbar c \pi^{2}}{240 a^{4}}\left[1+\frac{10}{21}\left(\frac{2 \pi \delta}{a}\right)^{2}+\right. \\
& \left.+\frac{1}{4}\left(\frac{2 \pi \delta}{a}\right)^{4}+\ldots\right],
\end{aligned}
$$

respectively.

We would like to emphasize, that if the conjecture of the previous paper [7] is physically correct, and so the resolution of measurement $\delta$ is a real physical parameter, which constraints maximal momenta of the field fluctuations, rather than being a formal regularization parameter, the deviations from the standard results should be observed if we compare two measurements with the same gap between planes, but different resolution.

In Fig. 2 below we present the comparison of the "exact" Casimir force between two plates in vacuum $(\delta=0)$, and that calculated according to (7) with $\delta / a=0.1$.

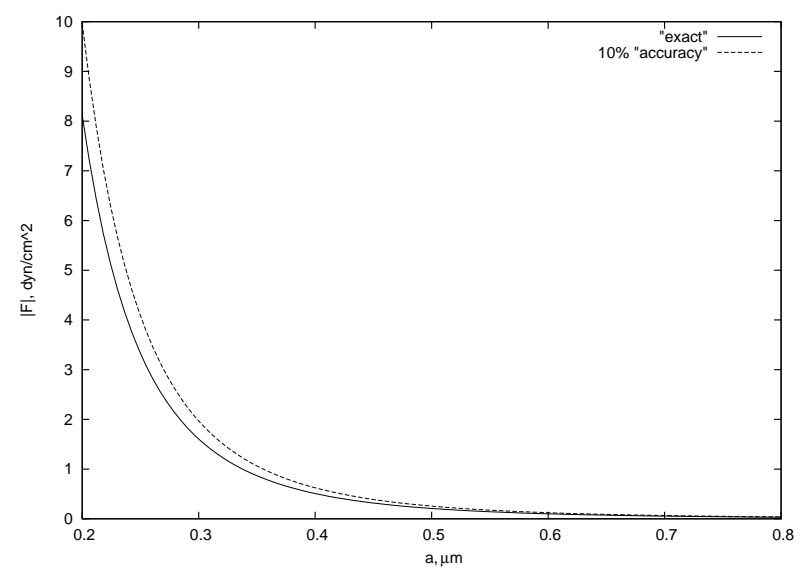

FIG. 2: Deviation of Casimir force between two plates of unit area in vacuum. The solid line corresponds to the "exact" Casimir force $(\delta=0)$, the dashed line corresponds to the scaledependent Casimir force with $\delta / a=0.1$

The dependence of the Casimir force on the cutoff parameter have been already suggested in the framework of the quantum field theory problem scaled to a condensed matter effective theory [9], where the inter-atomic distance plays the same role as the Planck length in high energy physics. It was concluded that actual Casimir force should be 
stronger than that predicted by conventional Casimir theory. The dependence on the cutoff scale also raises some criticism against the application of regularization methods to Casimir effect, specially for spherical geometry [10].

Interestingly, in present experiments the separation between plates is corrected by the factor $1+\left(\frac{\delta}{a}\right)^{2}$, derived from the Taylor expansion of the Casimir force, to account for the r.m.s. fluctuations of the random environment [11]. Our correction to the Casimir force due to the finite resolution of the measurement, given by Eq.(7) is also consistent with the limits posed by the precise measurement of the Casimir force given in [12] with the resolution $\delta / a \approx 4 \cdot 10^{-3}$. The choice of the scale parameter $\delta$ as a displacement amplitude is one of the possible simplifications. (Here we do not regard the dynamical effects [13].) For real experiments an important characteristics of the setup is a ratio of the boundary layer thickness to the distance between plates, which may be of order $h / a \sim 10^{-4} \div 10^{-2}$ [14]. With decreasing of the boundary plate thickness, according to the Lifshitz theory [15], the electron plasma of metal boundaries become utmost transparent for high frequency photons [16], and the ratio $h / a$ plays a role of another cutoff parameter.

An experimental study of the corrections to the Casimir force is certainly a challenging problem, where the dielectric permeability of the media should be taken into account at finite temperatures [4, 17, 18].

\section{Acknowledgments}

The authors are thankful to prof. N.V.Antonov and M.Z.Yuriev for useful discussions, and to prof. V.V.Nesterenko for critical comments. The research was supported in part by DFG Project 436 RUS 113/951 (M.V.A.) and by RFBR Project 11-02-00604-a and Programme of Creation and Development of the National University of Science and Technology "MISiS" (N.E.K.)
[1] H. Casimir, Proc. Kon. Ned. Akad. Wet. 51, 793 (1948).

[2] M. Sparnaay, Physica 24, 751 (1958).

[3] U. Mohideen and A. Roy, Physical Review Letters 81, 4549 (1998).

[4] G. Bressi, G. Carugno, R. Onofrio, and
G. Ruoso, Phys. Rev. Lett. 88, 041804 (2002).

[5] M. Bordag, U. Mohideen, and V. Mostepanenko, Phys. Rep. 353, 51 (2007).

[6] A. Rodriguez, F. Capasso, and S. Johnson, Nature Photonics 5, 211 (2011). 
[7] M. Altaisky, Phys. Rev. D 81, 125003 (2010).

[8] C. Itzykson and J.-P. Zuber, Quantum field theory (McGraw-Hill, Inc., 1980).

[9] G. Volovik, JETP Lett. 73, 375 (2001).

[10] C. Hagen, Phys. Rev. D 61, 065005 (2000).

[11] A. Sushkov, W. Kim, D. Dalvit, and S. Lamoreaux, Nature Physics 7, 230 (2011).

[12] R. S. Decca, D. López, E. Fischbach, G. L. Klimchitskaya, D. E. Krause, and V. M. Mostepanenko, Phys. Rev. D 75, 077101 (2007).

[13] A. Fedotov, N. Narozhny, and Y. Lozovik,
Journal of Optics B: Quantum and Semiclassical Optics 7, S64 (2005).

[14] V. Markov and Y. M. Pis'mak, J. Phys. A: Math. Gen. 39, 6525 (2006).

[15] E. Lifshitz, Sov. Phys. JETP 2, 73 (1956).

[16] N. Inui, Journal of the Physical Society of Japan 73, 332 (2004).

[17] V. Mostepanenko and N. Trunov, The Casimir Effect and its Applications (Oxford University Press, Oxford, 1997).

[18] V. Nesterenko, G. Lambiase, and G. Scarpetta, Riv. Nuovo Cim. 27, 1 (2004). 